\title{
SOME INTERESTING SPECIAL CASES OF A NON-LOCAL PROBLEM MODELLING OHMIC HEATING WITH VARIABLE THERMAL CONDUCTIVITY
}

\author{
D. E. TZANETIS AND P. M. VLAMOS \\ Department of Mathematics, Faculty of Applied Sciences, \\ National Technical University of Athens, \\ Zografou Campus, 15780 Athens, Greece (dtzan@math.ntua.gr)
}

(Received 7 February 2000)

Abstract The non-local equation

$$
u_{t}=\left(u^{3} u_{x}\right)_{x}+\frac{\lambda f(u)}{\left(\int_{-1}^{1} f(u) \mathrm{d} x\right)^{2}}
$$

is considered, subject to some initial and Dirichlet boundary conditions. Here $f$ is taken to be either $\exp \left(-s^{4}\right)$ or $H(1-s)$ with $H$ the Heaviside function, which are both decreasing. It is found that there exists a critical value $\lambda^{*}=2$, so that for $\lambda>\lambda^{*}$ there is no stationary solution and $u$ 'blows up' (in some sense). If $0<\lambda<\lambda^{*}$, there is a unique stationary solution which is asymptotically stable and the solution of the IBVP is global in time.

Keywords: non-local parabolic equations; blow-up; stability; local and global existence; stationary solutions

AMS 2000 Mathematics subject classification: Primary 35B30; 35B35; 35B40; 35K20; 35K55; 35K99

\section{Introduction}

Two non-local problems of significant practical interest $[\mathbf{1 3}]$ are considered, for which we study the asymptotic behaviour and blow-up of solutions.

The first initial boundary-value problem is

$$
\left.\begin{array}{ll}
u_{t}=\left(u^{3} u_{x}\right)_{x}+\frac{\lambda \exp \left(-u^{4}\right)}{\left(\int_{-1}^{1} \exp \left(-u^{4}\right) \mathrm{d} x\right)^{2}}, & -1<x<1, \quad t>0, \\
u(-1, t)=u(1, t)=0, & t>0, \\
u(x, 0)=u_{0}(x), & -1<x<1 ;
\end{array}\right\}
$$


while the second is

$$
\left.\begin{array}{ll}
u_{t}=\left(u^{3} u_{x}\right)_{x}+\frac{\lambda H(1-u)}{\left(\int_{-1}^{1} H(1-u) \mathrm{d} x\right)^{2}}, & -1<x<1, \quad t>0, \\
u(-1, t)=u(1, t)=0, & t>0, \\
u(x, 0)=u_{0}(x), & -1<x<1 .
\end{array}\right\}
$$

The parameter $\lambda$ is positive and $u$ mainly represents the dimensionless temperature. The nonlinear source terms are referred to as non-local because of the integral in the denominator. This kind of non-local term arises in many situations, especially when electric current flows through a conductor and ohmic heating is produced. The electrical resistance of the electric current is denoted by the Lipschitz continuous function $f(s)=$ $\exp \left(-s^{4}\right)>0$ or $f(s)=H(1-s) \geqslant 0$, for $s \geqslant 0$, which are both decreasing functions. For the derivation of the model of this non-local problem, we refer to $[\mathbf{2}, \mathbf{3}, \mathbf{8}, \mathbf{9}, \mathbf{1 3}]$ and the references cited therein. The above problems appear when the plasma is heated with ohmic heating, resulting in extremely high temperatures. As the plasma's temperature rises, the electrical resistance decreases and the ohmic heating becomes less effective, due to the fact that the denominator decreases faster than the numerator. It is this that brings about blow-up.

The diffusion term $u^{3}$ accounts for heat transport dominated by thermal radiation. The third power $\left(u^{3} u_{x}\right)_{x}$, or the fourth in the case of $\left(u^{4}\right)_{x x}$, comes from assuming the Stefan-Boltzmann law for emission of thermal radiation (see also $[\mathbf{1 0}, \mathbf{1 3}]$ ).

Problem (1.2) describes the case when the plasma loses all the electrical resistance as the temperature $u$ reaches the value 1 (by using a proper scaling, see [13]). Here $H(1-s)$ is the Heaviside function, which is neither Lipschitz nor strictly positive. The significance of the positivity and Lipschitz continuity of $f$ is related to the use of Picardtype arguments for proving the local existence of the solution $u$ in both problems (see $\S \S 2$ and 3$)$.

The corresponding steady problems of both problems (1.1) and (1.2) can be treated with quadratures. This is an additional reason for choosing $f(s)=\exp \left(-s^{4}\right)$ for problem (1.1). Thus we can find the steady solutions and the corresponding response diagrams explicitly. Also we obtain exact upper and lower solutions of the time-dependent problem. This fact is of great practical interest and differs totally from the methods used in the general case $[\mathbf{1 3}]$.

We also examine the existence and multiplicity of solutions for the steady problem, since this is the key to studying problem (1.1). The choice of decreasing $f$, apart from the practical interest, allows us to use comparison techniques.

Lacey $[\mathbf{8}, \mathbf{9}]$ had considered a similar problem with the standard linear diffusion term of the form $u_{x x}$. He found that there exists a critical value of the parameter $\lambda$, say $\lambda^{*}$, such that for $\lambda>\lambda^{*}$ there is no stationary solution and $u$ blows up, while for $\lambda<\lambda^{*}$ there exist stationary solutions and, if they are unique, then they are asymptotically stable and, moreover, $u$ is a global solution. Although the results obtained here are similar to those in $[\mathbf{8}, \mathbf{9}]$, there are a number of new technical points. 
This article has been organized as follows; in $\S 2$ we examine the exponential case, while in $\S 3$ the Heaviside function is considered.

\section{The exponential case $f(s)=\exp \left(-s^{4}\right)$}

In this case the initial boundary-value problem (1.1) takes the form

$$
\left.\begin{array}{ll}
u_{t}=\left(u^{3} u_{x}\right)_{x}+\frac{\lambda \exp \left(-u^{4}\right)}{\left(\int_{-1}^{1} \exp \left(-u^{4}\right) \mathrm{d} x\right)^{2}}, & -1<x<1, \quad t>0, \\
u(-1, t)=u(1, t)=0, & t>0, \\
u(x, 0)=u_{0}(x), & -1<x<1,
\end{array}\right\}
$$

where $u=u(x, t)$. The corresponding steady problem is

$$
\left.\begin{array}{c}
\left(w^{3} w^{\prime}\right)^{\prime}+\mu \exp \left(-w^{4}\right)=0, \quad-1<x<1, \\
w(-1)=w(1)=0
\end{array}\right\}
$$

where

$$
\mu=\frac{\lambda}{\left(\int_{-1}^{1} \exp \left(-w^{4}\right) \mathrm{d} x\right)^{2}} .
$$

The above boundary conditions might be replaced by something more general so that $w(-1)=\beta_{1}, w(1)=\beta_{2}$ with $\beta_{1} \neq \beta_{2}$, i.e. asymmetric, but for simplicity we take $\beta_{1}=\beta_{2}=0$. We choose $f(s)=\exp \left(-s^{4}\right)$ (decreasing) so as to be able to obtain the steady solutions of $(2.2)$ by quadratures.

The local existence and uniqueness of solutions of (1.1) can be proved by using Picardtype arguments, since $f(s)=\exp \left(-s^{4}\right)$ is positive and Lipschitz continuous. One can also use comparison techniques, since $f(s)=\exp \left(-s^{4}\right)$ is a decreasing function and a maximum principle holds (see $[\mathbf{8}, \mathbf{1 1}, \mathbf{1 3}]$ ).

\subsection{Stationary solutions}

Setting $w=W^{1 / 4},(2.2)$ becomes

$$
\left.\begin{array}{r}
W^{\prime \prime}+\nu \exp (-W)=0, \\
W(-1)=W(1)=0,
\end{array}\right\}
$$

where

$$
\nu=4 \mu=\frac{4 \lambda}{\left(\int_{-1}^{1} \exp \left(-w^{4}\right) \mathrm{d} x\right)^{2}} .
$$

Problem (2.3) has a unique solution $W(x)=2 \ln [\cos a x / \cos a]$, and $w(x)=$ $(2 \ln [\cos a x / \cos a])^{1 / 4}$, with $\mu=\frac{1}{4} \nu=\frac{1}{2} a^{2} \sec ^{2} a=\frac{1}{2} a^{2} \exp \left(M^{4}\right), 0<a<\frac{1}{2} \pi, M=w(0)$ (see $[\mathbf{3}, \mathbf{8}])$. Then $\lambda=\mu I^{2}(w)=\frac{1}{4} \nu I^{2}(w)=2 \sin ^{2} a<2$, where $I(w)=\left(\int_{-1}^{1} f(w) \mathrm{d} x\right)$. Since $\|w\|_{\infty}=w(0)=M=\left(\ln \left(\sec ^{2} a\right)\right)^{1 / 4}$, then $\lambda=\lambda(M)=2\left(1-\exp \left(-M^{4}\right)\right)$, $\lambda^{\prime}(M)=8 M^{3} \exp \left(-M^{4}\right)>0$, giving $\lambda \rightarrow 0$ as $M \rightarrow 0+($ or as $a \rightarrow 0), \lambda \rightarrow 2=\lambda^{*}-$ 

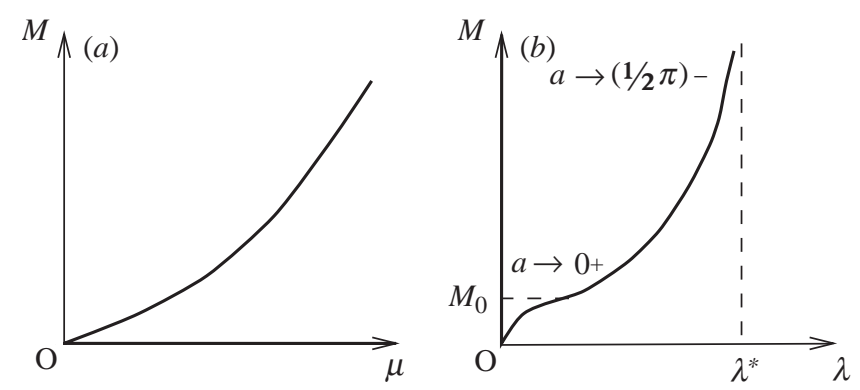

Figure 1. The response diagram of $f(s)=\exp \left(-s^{4}\right)$.

(a) Local diagram and $(b)$ non-local diagram.

as $M \rightarrow \infty$ (or as $a \rightarrow\left(\frac{1}{2} \pi\right)-$ ). Also, $\lambda^{\prime \prime}(M)=8 M^{2}\left(3-4 M^{4}\right) \exp \left(-M^{4}\right), \lambda^{\prime \prime}\left(M_{0}\right)=0$ for $M_{0}=\left(\frac{3}{4}\right)^{1 / 4}$, and we use the sign of $\lambda^{\prime \prime}(M)$ just for the convexity of the response diagram (see Figure 1). Moreover, $w_{\mu} \geqslant 0$ by using the usual maximum principle.

To each $M$ there corresponds a unique solution, this follows from a shooting argument. Also we remark that the maximum is taken at $x=0$ (because of the symmetry of the boundary conditions).

The above discussion implies the response diagram of Figure 1.

This non-degenerate example gives a critical value $\lambda^{*}=2$ so that for $0<\lambda<\lambda^{*}$ we have a unique solution of (2.2), whereas for $\lambda \geqslant \lambda^{*}$ there is no solution. Moreover, if $\lambda \rightarrow \lambda^{*}-$, or equivalently $a \rightarrow\left(\frac{1}{2} \pi\right)-$, then the solution $w(x)=W^{1 / 4}(x) \rightarrow \infty$ for all $x \in(-1,1)$.

\subsection{Stability for $\lambda<\lambda^{*}$}

We use comparison techniques: upper (lower) solutions decreasing (increasing) in time (see $[\mathbf{8}, \mathbf{1 1}, \mathbf{1 2}])$. We consider as a candidate upper solution the function $V$ of the form $V(x, t)=w(x ; \bar{\mu}(t))=[W(x ; \bar{\mu}(t))]^{1 / 4}$, and $\bar{\mu}(t)=\frac{1}{2} \bar{a}^{2}(t) \sec ^{2} \bar{a}(t)$. Now

$$
\begin{aligned}
P(V):=V_{t} & -\left(V^{3} V_{x}\right)_{x}-\frac{\lambda \exp \left(-V^{4}\right)}{\left(\int_{-1}^{1} \exp \left(-V^{4}\right) \mathrm{d} x\right)^{2}}=\dot{\bar{a}} \frac{\tan \bar{a}-x \tan \bar{a} x}{2 V^{3}} \\
& +\frac{1}{4} \bar{a}^{2}\left(2 \sin ^{2} \bar{a}-\lambda\right) \operatorname{cosec}^{2} \bar{a} \sec ^{2} \bar{a} x \geqslant \frac{1}{2} \dot{\bar{a}} b(\bar{a})+\frac{1}{4} \bar{a}^{2}\left(2 \sin ^{2} \bar{a}-\lambda\right) \operatorname{cosec}^{2} \bar{a},
\end{aligned}
$$

and $\dot{\bar{a}}(t)<0$, where

$$
0<\Phi(x ; \bar{a})=\frac{\tan \bar{a}-x \tan \bar{a} x}{V^{3}}<\Phi(0 ; \bar{a}):=b(\bar{a})=\frac{\tan \bar{a}}{\left(\ln \left(\sec ^{2} \bar{a}\right)\right)^{3 / 4}}
$$

in $[0,1]$, since $\Phi_{x}(x ; \bar{a})<0$;

$$
\left(\Phi(x ; a)^{(4 / 3)}\right)^{\prime}=h(x ; a) g(x ; a), \quad \text { where } h(x ; a)<0 \quad \text { and } \quad g(x ; a)>g(1 ; a)=0,
$$

since

$$
g^{\prime}(x ; a)<0 \quad \text { with } g(x ; a)=\frac{3 a \tan a x(\tan a-x \tan a x)}{\tan a x+a x \sec ^{2} a x}-4 \ln \frac{\cos a x}{\cos a} .
$$


Moreover, $w(x ; \bar{\mu})$ corresponds to a $\bar{\lambda},(\bar{\lambda}=\bar{\lambda}(\bar{\mu}))$, with

$$
\bar{\lambda}(t)=2 \sin ^{2} \bar{a}(t)>\lambda .
$$

Also, $V( \pm 1, t)=0, V_{0}(x)=w(x ; \bar{\mu}(0))>u_{0}(x)$, by choosing $\bar{a}(0)$ large enough, since $\bar{\mu}^{\prime}(\bar{a})>0$. If $\bar{a}(t)$ is chosen to satisfy

$$
0<-\dot{\bar{a}}=\phi(\bar{a})=\frac{\bar{a}^{2}}{2 b(\bar{a})}(\bar{\lambda}(t)-\lambda) \operatorname{cosec}^{2} \bar{a},
$$

then $V(x, t)$ is a decreasing-in-time upper solution to the $u$-problem. Hence, as $t \rightarrow \infty$, $\bar{\lambda}(t) \rightarrow \lambda+,\left(\bar{\lambda}^{\prime}(t)<0\right)$, or equivalently $\bar{\mu}(t) \rightarrow \mu+$ or $\bar{a}(t) \rightarrow \sin ^{-1} \sqrt{\frac{1}{2} \lambda}+$, giving that $V \rightarrow w+$ uniformly.

In a similar manner we construct a lower solution increasing in time:

$$
z(x, t)=w(x ; \underline{\mu}(t))=[W(x ; \underline{\mu}(t))]^{1 / 4}, \quad \underline{\mu}(t)=\frac{1}{2} \underline{a}^{2}(t) \sec ^{2} \underline{a}(t) .
$$

Again we obtain

$$
P(z)=z_{t}-\left(z^{3} z_{x}\right)_{x}-\frac{\lambda \exp \left(-z^{4}\right)}{\left(\int_{-1}^{1} \exp \left(-z^{4}\right) \mathrm{d} x\right)^{2}} \leqslant \frac{1}{2} \underline{\dot{a}} b(\underline{a})+\frac{1}{4} \underline{a}^{2}\left(2 \sin ^{2} \underline{a}-\lambda\right) \operatorname{cosec}^{2} \underline{a}=0,
$$

provided that

$$
0<\underline{\dot{a}}=-\phi(\underline{a})=\frac{\underline{a}^{2}}{2 b(\underline{a})}(\lambda-\underline{\lambda}(t)) \operatorname{cosec}^{2} \underline{a}
$$

and $\lambda>\underline{\lambda}(t)$.

By taking $z( \pm 1, t)=0, z_{0}(x)=w(x ; \underline{\mu}(0))<u_{0}(x)$, and choosing $\underline{a}(0)$ small enough, then $z$ is an increasing lower solution to the $u$-problem with $\underline{a}(t) \rightarrow \sin ^{-1} \sqrt{\frac{1}{2} \lambda}-$, or equivalently $\underline{\lambda}(t) \rightarrow \lambda$ - and $\underline{\mu}(t) \rightarrow \mu-$, as $t \rightarrow \infty$, giving, again, that $z \rightarrow w-$ uniformly in $x$. Finally, we get that $z \leqslant u \leqslant V, u$ is a global-in-time solution to (2.1), and $u \rightarrow w$ as $t \rightarrow \infty$ uniformly in $x$, where $w$ is the unique steady state; $w$ is an asymptotically stable solution.

\subsection{Blow-up for $\lambda>\lambda^{*}$}

Firstly, by using the previous increasing-in-time lower solution $z$, we obtain that $z \rightarrow \infty$ as $t \rightarrow \infty$. This implies that $u$ is unbounded as $t \rightarrow t^{*} \leqslant \infty$. We shall show that $t^{*}<\infty$, i.e. $u$ blows up at a finite time $t^{*}<\infty$. We now look for a blowing-up lower solution $z$ of the form

$$
z(x, t)=\left[p \ln \left(\frac{\cos a x}{\cos a}\right)\right]^{1 / 4}, \quad 0 \leqslant a=a(t)<\frac{1}{2} \pi, \quad t \geqslant t_{1},
$$

for some $t_{1}$ so that $u \geqslant z$ at $t=t_{1}$ and $\dot{a}(t)>0 ; p>0$ is a parameter which is chosen later on. Note that due to the unboundedness of $u$ we can consider (if $u$ were not to blow up) $a$ close to $\frac{1}{2} \pi$ for $t \geqslant t_{1}$. 
Thus we have

$$
\begin{aligned}
P(z) & =p \dot{a} \frac{\tan a-x \tan a x}{4 z^{3}}+\frac{1}{4} p a^{2} \sec ^{2} a x-\frac{\lambda \sec ^{p} a \cdot \sec ^{p} a x}{\left(\int_{-1}^{1} \sec ^{p} a x \mathrm{~d} x\right)^{2}} \\
& \lesssim \frac{1}{4} p b(a) \dot{a}-\frac{1}{16} \pi^{2}\left[\lambda(p-1)^{2}-p\right] \sec ^{2-p} a \sec ^{p} a x
\end{aligned}
$$

by taking into account that $a$ is close to $\frac{1}{2} \pi\left(0<\frac{1}{2} \pi-a \ll 1\right)$,

$$
\int_{-1}^{1} \sec ^{p} a x \mathrm{~d} x \sim \frac{4 \sec ^{p-1} a}{\pi(p-1)}, \quad p>1,
$$

and that

$$
0<\frac{\tan a-x \tan a x}{z^{3}}=\left(\frac{2}{p}\right)^{3 / 4} \Phi(x ; a) \leqslant\left(\frac{2}{p}\right)^{3 / 4} \Phi(0 ; a)=\left(\frac{2}{p}\right)^{3 / 4} b(a), \quad \text { in }[0,1] .
$$

Then $P(z)$ becomes

$$
P(z) \leqslant \frac{1}{2}\left(\frac{1}{2} p\right)^{1 / 4} b(a) \dot{a}-\frac{1}{16} \pi^{2} \Lambda \sec ^{2-p} a,
$$

since sec $a x \geqslant 1$, and on choosing $1<p<2$, so that $\Lambda=\lambda(p-1)^{2}-p>0$ or

$$
\lambda>\frac{p}{(p-1)^{2}} \quad \text { for a fixed } \lambda>\lambda^{*}=2 .
$$

Hence for $\lambda>\lambda^{*}=2$, there exists $p \in(1,2), p$ close to 2 , so that $\Lambda>0$.

Then we get

$$
\begin{aligned}
P(z) & \lesssim \frac{1}{4} p b(a) \dot{a}-\frac{1}{16} \pi^{2} \Lambda\left(\frac{1}{2} \pi-a\right)^{p-2} \\
& =\frac{1}{4} p \frac{\tan a}{(\ln (1 / \cos a))^{3 / 4}}\left[\dot{a}-\frac{\pi \Lambda}{4 p} \frac{(\ln (1 / \cos a))^{3 / 4}}{\tan a}\left(\frac{1}{2} \pi-a\right)^{p-2}\right],
\end{aligned}
$$

since

$$
b(a)=\frac{\tan a}{\left(\ln \left(\sec ^{2} a\right)\right)^{3 / 4}} .
$$

Finally, $P(z) \leqslant 0$, provided that $a$ satisfies

$$
\dot{a}=k\left(\frac{1}{2} \pi-a\right)^{p-1}, \quad a\left(t_{1}\right)=a_{1},
$$

where $k=(\pi \Lambda / 4 p)\left(\ln \left(\sec ^{2} a_{1}\right)\right)^{3 / 4}$, for some $a_{1} \leqslant a$. Then $a$ is increasing and so is $\left(\ln \left(\sec ^{2} a\right)\right)^{3 / 4}$, so we may choose $a_{1}=a\left(t_{1}\right)$. Now $z$ blows up at a finite time because $a(t)$ reaches $\frac{1}{2} \pi$ at finite time $T^{*}$, where

$$
0<T^{*} \leqslant \frac{1}{k} \int_{\omega_{1}}^{\infty} \frac{\mathrm{d} s}{s^{3-p}}+t_{1}=\frac{\omega_{1}^{p-2}}{k(2-p)}+t_{1}<\infty .
$$

This implies that $z$ blows up uniformly in $(-1,1)$ and also $u$ blows up at $t^{*} \leqslant T^{*}<\infty$. This blow-up is global, i.e. $u \rightarrow \infty$ as $t \rightarrow t^{*}<\infty$ for all $x$ in $(-1,1)$ (see $\left.[\mathbf{8}, \mathbf{1 2}, 13]\right)$. 


\section{The Heaviside function}

We consider the Heaviside function, $f(s)=H(1-s): f(s)=1$ for $s<1, f(s)=0$ for $s \geqslant 1$; this is neither Lipschitz nor strictly positive. Also we assume that $0<u_{0}(x) \leqslant 1$ and, for simplicity, $u_{0}(-x)=u_{0}(x)$, with $u_{0}^{\prime}(x) \leqslant 0, x \in(0,1)$, and $u_{0}^{\prime}(x)$ bounded below. Now, by using the maximum principle, with such initial data, we obtain $u \leqslant 1$, and that $u(-x, t)=u(x, t)$ (this is the reason for restricting our attention to $0<x<1$ ).

Now, problem (1.2) becomes

$$
\left.\begin{array}{ll}
u_{t}=\left(u^{3} u_{x}\right)_{x}+\frac{\lambda H(1-u)}{\left(\int_{-1}^{1} H(1-u) \mathrm{d} x\right)^{2}}, & 0<x<1, \quad t>0, \\
u_{x}(0, t)=u(1, t)=0, & t>0, \\
u(x, 0)=u_{0}(x), & 0<x<1 .
\end{array}\right\}
$$

The local existence and uniqueness of solutions of (3.1) can be obtained by using a family of regularized problems of the form of (3.1) with Lipschitz nonlinearities $f_{\epsilon}(s)$. More precisely,

$$
f_{\epsilon}(s)=g\left(\frac{s-1}{\epsilon}\right)+\epsilon, \quad 0<\epsilon \ll 1,
$$

where $g(s)$ is a decreasing and Lipschitz continuous function in $\mathbb{R}$, such that $g(s)=0$ for $s \leqslant-1$ and $g(s)=1$ for $s \geqslant 1$, with $\lim _{\epsilon \rightarrow 0} f_{\epsilon}(s)=H(1-s)$ (for more details see $[6]$ ).

Hence $u_{t}=\left(u^{3} u_{x}\right)_{x}$, where $u \geqslant 1$ and $u_{t}=\left(u^{3} u_{x}\right)_{x}+\left(\lambda / m^{2}(t)\right)$, where $u<1$, with $m(t)$ the measure of the subset of $[-1,1]$ where $u<1$ so $m(t) \in(0,2], t>0$.

Thus either $u<1$, for all $x \in(0,1)$, in which case (3.1) becomes

$$
\left.\begin{array}{r}
u_{t}=\left(u^{3} u_{x}\right)_{x}+\frac{1}{4} \lambda, \quad 0<x<1, \quad t>0, \\
u_{x}(0, t)=0, \quad u(1, t)=0,
\end{array}\right\}
$$

or there exists some $s=s(t) \in(0,1)$ so that

$$
\left.\begin{array}{ll}
u_{t}=\left(u^{3} u_{x}\right)_{x}+\frac{\lambda}{4(1-s)^{2}}, & 0 \leqslant u<1, \quad s<x<1, \quad t>0, \\
u(x, t)=1, \quad u_{x}(x, t)=0, & \text { for } 0 \leqslant x \leqslant s, \quad t>0, \\
u(1, t)=0, & t>0 .
\end{array}\right\}
$$

The corresponding steady problems of (3.2), (3.3) are, respectively,

$$
\left.\begin{array}{c}
\left(w^{3} w^{\prime}\right)^{\prime}+\frac{1}{4} \lambda=0, \quad 0<x<1, \\
w^{\prime}(0)=0, \quad w(1)=0,
\end{array}\right\}
$$

and

$$
\left.\begin{array}{c}
\left(w^{3} w^{\prime}\right)^{\prime}+\frac{\lambda}{4(1-S)^{2}}=0, \quad S<x<1 \\
w^{\prime}(x)=0, \quad w(x)=1 \quad \text { for } 0 \leqslant x \leqslant S \\
w(1)=0 .
\end{array}\right\}
$$



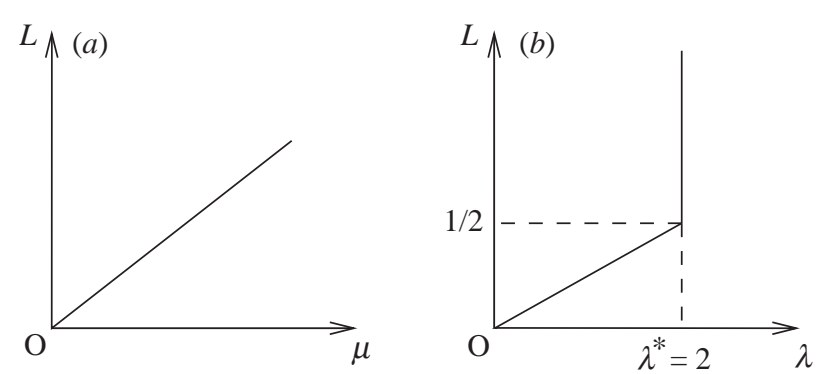

Figure 2. The response diagrams for the Heaviside function $L=-\lim _{x \rightarrow 1-} w^{3}(x) w^{\prime}(x)$.

(a) Local diagram and (b) non-local diagram.

The solution of (3.4) is $w(x)=\left[\frac{1}{2} \lambda\left(1-x^{2}\right)\right]^{1 / 4}=W(x)^{1 / 4}$ and $w<1$ if $\lambda<\lambda^{*}=2$, while the solution of $(3.5)$ is

$$
w(x ; S)=\left[1-\frac{(x-S)^{2}}{(1-S)^{2}}\right]^{1 / 4}=W^{1 / 4}(x ; S) \quad \text { for } S<x<1,
$$

and $w(x ; S)=1$ for $0 \leqslant x \leqslant S ; \lambda=\lambda^{*}$. If $\lambda>\lambda^{*}=2$, then there is no steady solution $w \leqslant 1$. Moreover,

$$
L= \begin{cases}\frac{1}{4} \lambda, & \text { for } \lambda<\lambda^{*}=2 \\ \frac{\lambda^{*}}{4(1-S)}, & \text { for } 0<S<1\end{cases}
$$

where $L=-\lim _{x \rightarrow 1-} w^{3}(x) w^{\prime}(x)$.

The above conclusions are summed up in Figure 2.

\subsection{Stability for $\lambda<\lambda^{*}$}

To study the stability of the steady solutions, we use upper and lower solutions as for the exponential case.

Let $\lambda<\lambda^{*}$; for any initial data $u_{0}(x)$ we can assume $S_{0}$ so that

$$
u_{0}(x) \leqslant 1 \quad \text { for } 0 \leqslant x \leqslant S_{0} \quad \text { and } \quad u_{0}(x) \leqslant w\left(x ; S_{0}\right) \text { for } S_{0} \leqslant x \leqslant 1 .
$$

We look for an upper solution which is decreasing in time and of the form

$$
\begin{aligned}
& V(x, t)=1, \quad V_{x}(x, t)=0, \quad 0 \leqslant x \leqslant s(t), \quad t>0, \\
& V(x, t)=w(x ; s(t)), \\
& V(1, t)=0 \text {, } \\
& \left.\begin{array}{l}
s(t)<x<1, \quad t>0, \\
t>0 .
\end{array}\right\}
\end{aligned}
$$

Then

$$
\begin{aligned}
P(V) & =\frac{(1-x)(x-s)}{2 w^{3}(1-s)^{3}} \dot{s}+\frac{\lambda^{*}-\lambda}{4(1-s)^{2}} \\
& \geqslant \frac{\dot{s}}{2(1-s)^{2}}+\frac{\lambda^{*}-\lambda}{4(1-s)^{2}}=0, \quad s<x<1, \quad 0<t<t_{1},
\end{aligned}
$$


provided that $s(t)$ satisfies $\dot{s}=-\frac{1}{2}\left(\lambda^{*}-\lambda\right)<0$. The latter gives that $s(t)=$ $-\frac{1}{2}\left(\lambda^{*}-\lambda\right) t+s_{0}$ for $0<t<t_{1}$, where $t_{1}=2 S_{0} /\left(\lambda^{*}-\lambda\right)$, i.e. $s\left(t_{1}\right)=0$. Inequality (3.7) is a consequence of the fact that $(1-x) / w^{3}=\phi(x ; S) \leqslant \phi(S+; S)=1-S \leqslant 1$, since $\mathrm{d} \phi / \mathrm{d} x<0$ (this can be easily proved). Also, in the interval $0 \leqslant x \leqslant s(t), 0<t<t_{1}$, $P(V)=0$.

For $t \geqslant t_{1}$ we look for an upper solution $V$ having the form $V(x, t)=w(x ; \bar{\lambda}(t))=$ $\left[\frac{1}{2} \bar{\lambda}(t)\left(1-x^{2}\right)\right]^{1 / 4}, 0<x<1, t \geqslant t_{1}$, where $\frac{1}{2} \bar{\lambda}\left(t_{1}\right)=1$. Then again

$$
P(V)=\frac{\left(1-x^{2}\right)}{8 w^{3}} \frac{\mathrm{d} \bar{\lambda}(t)}{\mathrm{d} t}+\frac{\bar{\lambda}(t)-\lambda}{4} \geqslant \frac{\dot{\bar{\lambda}}(t)}{4}+\frac{\bar{\lambda}(t)-\lambda}{4}=0
$$

since $(1-x) / w^{3}=\phi(x ; \lambda) \leqslant \phi(0 ; \lambda) \leqslant 1$, and provided that $\bar{\lambda}(t)$ satisfies $\dot{\bar{\lambda}}(t)=-(\bar{\lambda}(t)-$ $\lambda)<0, t \geqslant t_{1}$, giving $\bar{\lambda}(t)=\lambda+(2-\lambda) \exp \left(t_{1}-t\right), t \geqslant t_{1}$.

In any of the above cases $V( \pm 1, t)=0$. Hence $V(x, t)$ is an upper solution such that $V(x, t) \rightarrow w(x)+$, uniformly in $x$, as $t \rightarrow \infty$, since $\bar{\lambda}(t) \rightarrow \lambda<\lambda^{*}=2$ as $t \rightarrow \infty$.

In a similar manner we construct a lower solution

$$
z(x, t)=w(x ; \underline{\lambda}(t))=\left[\frac{1}{2} \underline{\lambda}(t)\left(1-x^{2}\right)\right]^{1 / 4}, \quad 0<x<1, \quad t>0 .
$$

Then $P(z) \leqslant 0$, provided again that $\underline{\lambda}(t)$ satisfies

$$
\underline{\dot{\lambda}}(t)=-(\underline{\lambda}(t)-\lambda)>0, \quad \underline{\lambda}(0)=0 .
$$

This gives $\underline{\lambda}(t)=\lambda(1-\exp (-t)), t \geqslant 0$, which implies that $\underline{\lambda}(t) \rightarrow \lambda$ as $t \rightarrow \infty$. Hence $z$ is an increasing lower solution such that $z(x, t) \rightarrow w(x)-$, uniformly in $x$, as $t \rightarrow \infty$.

Finally, if $0<\lambda<\lambda^{*}$, the unique solution $w=w(x ; \lambda)$ is asymptotically stable; moreover, $z \leqslant u \leqslant V, u$ is a global-in-time solution and $u(x, t) \rightarrow w(x)$, uniformly in $x$, as $t \rightarrow \infty$.

\section{2. 'Blow-up' for $\lambda>\lambda^{*}=2$}

In this subsection we prove that for $\lambda>\lambda^{*}=2, u$ 'blows up' in finite time. By 'blow-up' of $u$, in this case, we mean that $u$ becomes 1 in the whole interval $(-1,1)$ as $t \rightarrow t^{*}<\infty$ (we recall that $u \leqslant 1$ ). This means that the mathematical model fails to describe the physical problem near to the 'blow-up' time.

Assuming that $u$ does not 'blow up', then $u$ exists for all $t>0$. We can construct a lower solution $z(x, t)=w(x ; \underline{\lambda}(t))=\left[\frac{1}{2} \underline{\lambda}(t)\left(1-x^{2}\right)\right]^{1 / 4}$ with $\underline{\lambda}(0)=0, z( \pm 1, t)=0$ and $z(x ; 0) \leqslant u_{0}(x)$ in $[-1,1]$. As in $\S 3.1$, there exists a $t_{1}$ such that $\frac{1}{2} \underline{\lambda}\left(t_{1}\right)=1$.

For $t \geqslant t_{1}$ we introduce the following form for $z: z(x, t)=1,0 \leqslant x \leqslant s$,

$$
z(x, t)=w(x ; s(t))=\left[1-\frac{(x-s(t))^{2}}{(1-s(t))^{2}}\right]^{1 / 4},
$$

$s(t)<x<1, t \geqslant t_{1}$, and satisfying (3.4), with $s\left(t_{1}\right)=0$. Then

$$
P(z)=\frac{(1-x)(x-s)}{2 w^{3}(1-s)^{3}} \dot{s}+\frac{\lambda^{*}-\lambda}{4(1-s)^{2}} \leqslant \frac{\dot{s}}{2(1-s)^{2}}+\frac{\lambda^{*}-\lambda}{4(1-s)^{2}}=0,
$$


provided that $s(t)$ satisfies

$$
\dot{s}=\frac{1}{2}\left(\lambda-\lambda^{*}\right)>0, \quad t \geqslant t_{1} \quad \text { and } \quad s\left(t_{1}\right)=1 .
$$

Thus $s(t)=\frac{1}{2}\left(\lambda-\lambda^{*}\right)\left(t-t_{1}\right), t \geqslant t_{1}$, but then $s\left(T^{*}\right)=1$ as $T^{*}=2 /\left(\lambda-\lambda^{*}\right)+t_{1}<\infty$.

This implies that $z(\cdot, t) \rightarrow 1$ for all $x$ in $(-1,1)$ as $t \rightarrow T^{*}<\infty$. Since $z$ is a lower solution, $z \leqslant u, u$ also becomes 1 in $(-1,1)$ as $t \rightarrow t^{*} \leqslant T^{*}<\infty$, and a sort of 'blow-up' has been established with derivatives becoming unbounded at $x=-1,1$ (see also $[8,9,12])$.

\section{Discussion}

In this paper we have examined a problem having some special non-local, although interesting, source terms and a variable conduction term. The conduction term is of the form $\left(u^{3} u_{x}\right)_{x}$ and comes from the Stefan-Boltzmann law for emission of thermal radiation. Since $f$, in any case, is decreasing, we can use comparison methods. It has been proved that there exists a critical value $\lambda^{*}=2$ such that for $\lambda>\lambda^{*}$ there is no stationary solution, while the time-dependent solution 'blows up' globally. For $0<\lambda<\lambda^{*}$ the unique steady solution is globally asymptotically stable. Other boundary conditions can be tackled similarly (see $[\mathbf{8}, \mathbf{1 3}]$ ).

The above results can be extended straightforwardly to other terms such as $\left(u^{n} u_{x}\right)_{x}$, for $n \in N^{*}$.

Acknowledgements. The authors thank Dr A. A. Lacey for several fruitful discussions. This work was partly supported by The National Hellenic Research Foundation (EIE).

\section{References}

1. J. Bebernes AND D. EBerly, Mathematical problems from combustion theory, Applied Mathematical Sciences, vol. 83 (Springer, 1989).

2. J. W. Bebernes And A. A. LACEy, Global existence and finite-time blow-up for a class of nonlocal parabolic problems, Adv. Diff. Eqns 2 (1997), 927-953.

3. J. A. CARRILlo, On a non-local elliptic equation with decreasing nonlinearity arising in plasma physics and heat conduction, Nonlinear Analysis TMA 32 (1998), 97-115.

4. G. Cimatтi, Remark on existence and uniqueness for the thermistor problem under mixed boundary conditions, Q. Appl. Math. 47 (1989), 117-121.

5. G. Cimatti, The stationary thermistor problem with a current limiting device, Proc. $R$. Soc. Edinb. A 116 (1990), 79-84.

6. N. I. Kavallaris And D. E. Tzanetis, A nonlocal diffusion-convection problem for the Heaviside function in ohmic heating process, Diff. Integ. Eqns, in press.

7. H. B. Keller And D. S. Cohen, Some positone problems suggested by nonlinear heat generation, J. Math. Mech. 16 (1967), 1361-1367.

8. A. A. LACEY, Thermal runaway in a nonlocal problem modelling ohmic heating, Part I, Model derivation and some special cases, Eur. J. Appl. Math. 6 (1995), 127-144.

9. A. A. LACEY, Thermal runaway in a nonlocal problem modelling ohmic heating, Part II, General proof of blow-up and asymptotic of runaway, Eur. J. Appl. Math. 6 (1995), 201224 . 
10. Mathematical Study Group with Industry Report (Birmingham, 1990).

11. D. H. SATtinger, Monotone methods in nonlinear elliptic and parabolic boundary value problems, Indiana Univ. Math. J. 21 (1972), 979-1000.

12. D. E. TzAnETis, Blow-up of radially symmetric solutions of a non-local problem modelling ohmic heating (1999), preprint.

13. D. E. Tzanetis And P. M. Vlamos, A nonlocal problem modelling ohmic heating with variable thermal conductivity (1999), Nonlinear Analysis: Real World Appl., in press. 